\title{
基于多点力约束的视网膜手术机器人的导纳控制
}

\author{
贺昌岩 杨 洋 \\ (北京航空航天大学机械工程及自动化学院 北京 100191)
}

\begin{abstract}
摘要: 在视网膜血管注药手术中, 手术器械与眼球巩膜刺入孔和视网膜目标血管处产生接触。为保证手术过程中这两处组织 的受力在安全阈值内, 提出了一种基于多点接触力的机器人约束控制算法。以手术器械轴部与眼球巩膜的接触力(巩膜力)和 器械尖端与视网膜血管的接触力(尖端力)为输入, 设计了机器人的导纳控制器。导纳控制器输出机器人坐标系下器械尖端处 及巩膜接触位置的速度。这两处的运动速度在转换到同一坐标系下后对机器人进行运动约束。在通过导纳算法控制机器人的 速度时, 提出了一种非线性速度轨迹规划方法, 实现速度的平滑变化。所提出的约束控制算法在硅胶眼球模型上进行了模拟 视网膜注药试验, 试验结果显示在外部扰动存在的情况下, 巩膜力和尖端力均被保持在给定阈值之内, 表明了所提出的多点 力约束机器人控制算法对提高手术安全方面的有效性。
\end{abstract}

关键词: 导纳控制; 运动约束; 机器人辅助视网膜手术; 微力感知控制

中图分类号: TG156

\section{Multipoint Force-constrained Admittance Control for Retinal Surgical Robot}

\author{
HE Changyan YANG Yang
}

(School of Mechanical Engineering and Automation, Beihang University, Beijing 100191)

\begin{abstract}
In retinal vein cannulation surgery, the surgical instrument needs to collide with the eyeball sclera incision and the target retinal vessel. In order to keep the tool-tissue forces at these two spots being within safety thresholds during the surgery, a robotic constrained control algorithm based on multipoint contact force is proposed. A robotic admittance controller was designed with the inputs of the contact forces between the surgical instrument shaft and the eyeball sclera (scleral force), and between the instrument tip and the retinal vessels (tip force). The admittance controller outputs the velocities of the instrument at the tip and at the sclera port in the robot coordinate system. The velocities of these two places are used to restrict the movement of the robot after being transformed into the same coordinate system. In the calculation of the velocities, a non-linear velocity trajectory planning method is proposed to achieve smooth changes of the velocity. The proposed constrained control algorithm was performed on a silicone eyeball model to simulate retinal injection experiments. The experimental results showed that in the presence of external disturbances, both the scleral force and the tip force are kept within given thresholds, which verified the effectiveness of multipoint force-constrained robot control algorithms in improving surgery safety.
\end{abstract}

Key words: admittance control; motion constraint; robot-assisted retinal surgery; micro-force sensing and control

\section{0 前言}

眼科手术是一项极为精密的手术操作, 对于医 生的手术技能和经验有极高的要求。眼科手术的 挑战性主要表现在: (1) 手术空间狭小, 眼球为一 个平均直径仅有 $23 \mathrm{~mm}$ 的球体, 所有手术操作均

* 国家自然科学基金(51875011)和国家重点研发计划(2017YFB1302702) 资助项目。20200428 收到初稿, 20200716 收到修改稿
需要在此空间中完成; (2) 手术操作力尺度微小, 甚至超过人手的感知极限 ${ }^{[1]}$, 医生很难精确感知器 械与眼组织的接触力 ${ }^{[2]}$; (3) 手术操作精度要求很 高, 而人手的颤抖使得操作很难进行。除此之外, 在如图 1 所示的血管注药等眼内手术操作 ${ }^{[3]}$ 中, 医生通常需要把持手术器械穿过固定在眼球巩膜 开口处的套管进入眼内, 然后控制器械尖端对眼 底视网膜组织进行操作。此操作过程中眼球同时 在巩膜开口及眼底视网膜两处位置受到器械施加 
的作用力, 也就是说, 手术器械的运动受到多点 接触力的约束。

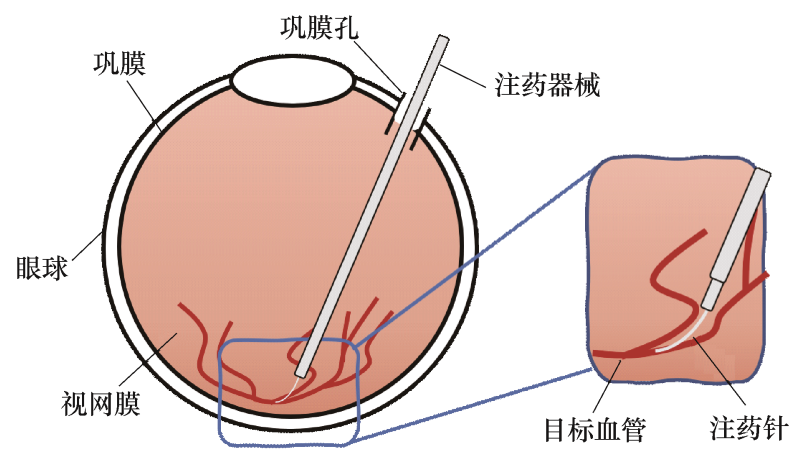

图 1 眼底视网膜血管注药手术操作示意图

为降低眼科手术对医生手术技能的苛刻要求, 缓解或解决上述挑战, 一系列构型各异用于眼科 手术操作的机器人系统被研制了出来 ${ }^{[4]}$ 。机器人具 有运动精度高、操作稳定等特点, 可以提高和拓 展医生的手术技能, 提高手术效果。总体来看, 眼科手术机器人可以分为四大类, 包括: (1) 以约 翰霍普金斯大学提出的稳定手机器人(Steady hand eye robot, SHER $)^{[5]}$ 为代表的协同控制类机器人, 该 机器人允许医生直接把持机器人的末端对机器人 进行操纵; (2) 以埃因霍温大学研制的 Preceyes 系 统 $^{[6]}$ 、鲁汶大学研制的视网膜机器人 ${ }^{[7]}$ 、北京航空 航天大学研制的双臂眼内手术机器人 ${ }^{[8]}$ 、慕尼黑工 业大学提出的紧凑型并联机器人 ${ }^{[9]}$ 、哥伦比亚大学 研制的半圆弧导轨机器人 ${ }^{[10]}$ 、加州大学洛杉矶分 校研制的眼科手术机器人 ${ }^{[11]}$ 以及东京大学研制的 并联手术机器人系统 ${ }^{[12]}$ 为代表的主从式控制类机 器人, 该类机器人通过使用单独的主手供医生操 作, 从机械臂执行手术动作; (3) 以卡耐基梅隆大 学提出的 Micron 系统 ${ }^{[13]}$ 为代表的手持式机器人 等, Micron 机器人构型小巧, 可以较好地适应不 同的手术环境, 比如野外或临时手术; (4) 以 $\mathrm{HE}$ 等 ${ }^{[14]}$ 提出的 Snake Robot、HUBSCHMAN 等 ${ }^{[15]}$ 提 出的 Micro Hand、KRATOCHVIL 等 ${ }^{[16]}$ 提出的 OctMag 磁场驱动机器人等为代表的灵巧操作器, 该类末端器可延伸机器人在眼内的灵活性, 增加 机器人的末端自由度。

上述眼科手术机器人系统的效用主要集中在帮 助医生过滤手部颤抖、提高手术精度等, 对于器械 在眼内的约束运动却尚少涉及。为了避免过大的器 械作用力对眼球组织造成挤压与损伤, 手术过程中 需要同时对器械与巩膜的交互力(巩膜力) 和器械与 视网膜组织的交互力(尖端力)进行控制, 保证眼球 组织受力在一定的安全阈值之内。另外, 手术过程
中病人的眼球会不自主地产生不规律的转动, 转动 会导致眼组织(巩膜开口及视网膜处)受到额外的器 械作用力, 手术过程中也需要对这种外部扰动力进 行抑制。肖晶晶等 ${ }^{[17]}$ 提出了一种基于多刺入点的视 网膜手术机器人的约束运动算法, 该算法考虑了多 个器械在眼内的运动规划和虚拟约束, 但是只进行 了模拟仿真, 在真实环境下的效用尚待验证。 EBRAHIMI 等 ${ }^{[18-19]}$ 提出了一种自适应控制算法, 可 以对眼球组织的刚性进行在线估计并对器械与眼 球的交互力进行实时控制，但是该算法只考虑了 器械与巩膜的交互, 尚未涉及器械与眼底视网膜 组织的交互。HE 等 ${ }^{[20]}$ 将器械与巩膜的交互力作为 输入, 设计了一种非线性的导纳控制算法, 可以 维持器械在眼内进行手术操作时不发生过大变 形, 同样, 该算法对于器械与眼底视网膜组织的 交互并未涉及。

基于此, 本文研究了眼内手术中多点力约束下 手术机器人的运动控制。首先基于巩膜力和尖端 力提出了一种力驱动的约束控制算法, 并设计了 机器人的导纳控制器。巩膜力和尖端力分别被用 来约束器械轴部和器械尖端处的运动, 其作为导 纳控制器的输入, 计算机器人坐标系下器械尖端 处及巩膜接触位置的速度。为实现速度的平滑变 化, 在导纳控制器中, 提出了一种非线性的速度 轨迹规划方法。最后, 利用所提出的约束控制算 法, 本文在硅胶眼球模型上进行了模拟视网膜注 药试验, 试验结果显示在外部扰动存在的情况下, 巩膜力和尖端力均被保持在给定阈值之内, 从而 表明了所提多点力约束机器人控制算法可以在一 定程度上提高手术安全性。

\section{1 视网膜手术机器人硬件系统}

\section{1 手术机器人系统}

本文使用了 SHER 机器人 ${ }^{[5]}$ 作为手术机器人 平台, 如图 2 所示。SHER 机器人采用人机协同 的操作方式, 此操作方式的特点在于医生直接握 持机器人末端的手术器械, 医生的操纵力通过机 器人末端的 6 轴力传感器传递到机器人控制系统。 机器人控制器根据医生的操作力计算并输出机器 人的运动速度, 进而控制机器人实现相应的运动, 如式(1)所示

$$
\boldsymbol{v}=\alpha \boldsymbol{F}
$$

式中, $\boldsymbol{v} \in \mathbf{R}^{6 \times 1}$ 表示机器人的运动速度, $\boldsymbol{F} \in \mathbf{R}^{6 \times 1}$ 表 示医生对机器人的操作力, $a$ 表示映射系数。 


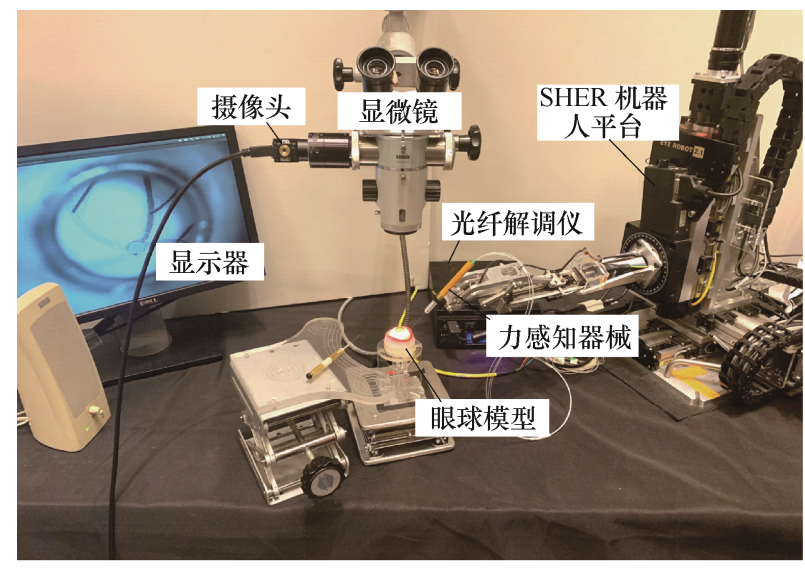

图 2 手术机器人系统

这种控制方式为医生提供了更直观的操作方 式, 并利于医生缩短学习曲线。SHER 机器人由 3 个平移台和 1 个由平行四杆组成的远程运动中心机 构组成, 具有 5 个自由度, 包括 3 个平移和 2 个绕 远程运动中心点 $(\mathrm{RCM})$ 的旋转自由度, 其平移运动 精度可达 $3 \mu \mathrm{m}$, 旋转精度可达 $0.005^{\circ}$ 。

\section{2 力感知手术器械}

为对巩膜力和尖端力进行感知, 本文使用了本 课题组之前研制的多自由度力感知视网膜血管注药 器 ${ }^{[21]}$, 如图 3 所示。力感知器械基于光纤传感器实 现感知功能, 3 根光纤管平行布置, 沿轴向相隔 $120^{\circ}$ 贴附在注药器械轴的表面, 每根光纤管内置有 3 组布拉格光纤光柱(Fiber Bragg grating, FBG)。FBG 能够对微小应变进行响应, 其反射波长与所受的局 部应变成线性关系。基于此, 当器械受力变形时,

FBG 光纤将受到微小应变, 从而导致 FBG 的返回 波长发生变化。此时, FBG 的波长差与器械的受力 有以下关系

$$
\Delta \boldsymbol{S}=\boldsymbol{K} \boldsymbol{F}
$$

式中, $\Delta \boldsymbol{S}$ 表示 $\mathrm{FBG}$ 的波长差, $\boldsymbol{K}$ 表示常数映射矩 阵, $\boldsymbol{F}$ 表示器械的受力。

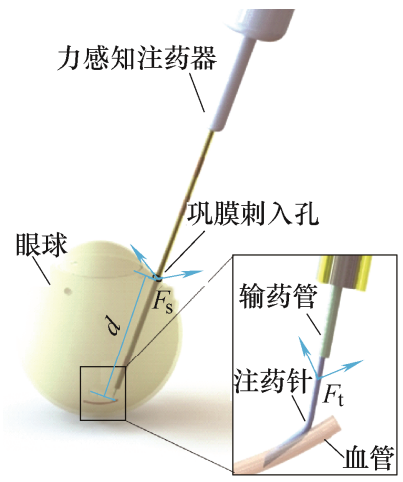

(a)

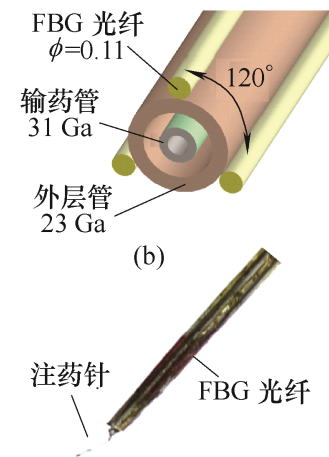

(c)
图 3 力感知视网膜血管注药器械
由式(2)可进一步解算出器械的巩膜力 (记为 $\boldsymbol{F}_{s}$ )、尖端力 (记为 $\boldsymbol{F}_{t}$ ) 以及器械在眼内的深度(记为 $d)$ 。经过标定之后, 力感知注药器的巩膜力、尖端 力和深度的感知均方根误差分别为 $1.5 \mathrm{mN} 、 0.7 \mathrm{mN}$ 和 $0.3 \mathrm{~mm}$ 。

注药器结构包括 $23 \mathrm{Ga}(\Phi=0.6 \mathrm{~mm})$ 的外层管、 $31 \mathrm{Ga}(\Phi=0.4 \mathrm{~mm})$ 的输药管以及位于尖端用于刺入 血管的 $36 \mathrm{Ga}(\Phi=0.1 \mathrm{~mm})$ 的注药针。为了便于刺入 血管, 注药针被弯折与器械轴线成 $30^{\circ}$ 夹角。

\section{2 多点接触力约束控制算法}

由上文可知, 多点力约束控制算法的主要目标 为控制眼球巩膜处与视网膜处所受到的器械作用力 不超过给定的阈值。基于前文设计并研制的力感 知器械, 手术过程中巩膜力 $F_{s}$ 、尖端力 $F_{t}$ 以及深 度 $d$ 均可以实时获得。将此三项感知量作为输入 与反馈, 设计机器人的导纳控制算法, 计算并调 整机器人的运动速度, 从而实现对以上器械与眼 组织作用力的控制。由此得到的控制算法框图如 图 4 所示。

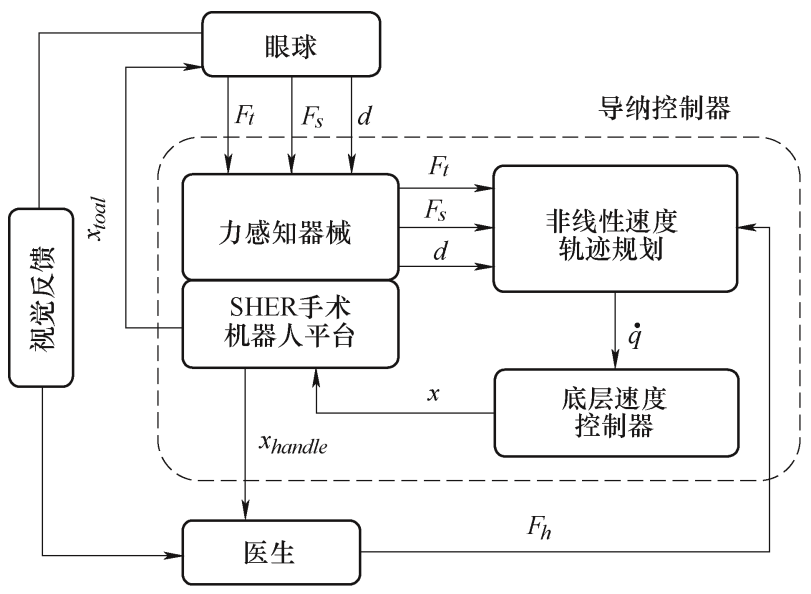

图 4 多点接触力约束控制框图

\section{1 基本导纳控制方式}

本文使用的手术机器人为 SHER 机器人平台, 如前文所述, 机器人工作时受医生的直接操作, 即 医生用手把持机器人的末端器, 产生的操作力经过 计算后得到机器人的运动速度, 如式(3)和式(4)所示

$$
\begin{gathered}
\dot{\boldsymbol{x}}_{h h}=\alpha \boldsymbol{F}_{h h} \\
\dot{\boldsymbol{x}}_{r h}=\boldsymbol{A} \boldsymbol{d}_{g_{r h}} \dot{\boldsymbol{x}}_{h h}
\end{gathered}
$$

式中, $\dot{\boldsymbol{x}}_{h h}$ 和 $\dot{\boldsymbol{x}}_{r h}$ 分别为在器械坐标系 $\{h\}$ 及机器人 坐标系 $\{r\}$ (图 5)中机器人器械的运动速度, $\boldsymbol{F}_{h h}$ 为操 作者施加在器械上的操作力在器械坐标系 $\{h\}$ 中的 
表达, $a$ 表示增益系数, 可通过机器人的脚踏板进 行调节, $\boldsymbol{A d}_{g_{r h}}$ 表示器械坐标系 $\{h\}$ 到机器人坐标系 $\{r\}$ 的伴随变换矩阵, 可以进一步写为

$$
\boldsymbol{A d}_{g_{r h}}=\left[\begin{array}{cc}
\boldsymbol{R}_{r h} & \hat{\boldsymbol{p}}_{r h} \boldsymbol{R}_{r h} \\
0 & \boldsymbol{R}_{r h}
\end{array}\right]
$$

式中, $\boldsymbol{R}_{r h}$ 和 $\boldsymbol{p}_{r h}$ 分别表示器械坐标系到机器人坐标 系的旋转矩阵及平移矢量, $\hat{\boldsymbol{p}}_{r h}$ 表示 $\boldsymbol{p}_{r h}$ 的反对称 矩阵。

\section{2 巩膜力约束控制}

在巩膜力约束控制上, 将巩膜力作为输入计算 机器人末端的器械在巩膜坐标系 $\{s\}$ 下的平移速度 $\left(v_{s x}, v_{s y}\right)$, 通过对平移速度的控制来抑制巩膜力的 大小, 如式(6)所示

$$
\boldsymbol{v}_{s i}= \begin{cases}A \sin \left(B \Delta \boldsymbol{F}_{s i}\right) & 0<\Delta F_{s i} \leqslant \max \Delta F_{s} \\ \operatorname{sgn}\left(\boldsymbol{F}_{s i}\right) \max v_{s} & \Delta F_{s i}>\max \Delta F_{s} \\ 0 \quad \text { 其他 } & \end{cases}
$$

式中, $\Delta F_{s i}=\left|F_{s i}\right|-F_{s}^{*}$ 表示巩膜力与安全阈值的差值, 其中 $\boldsymbol{F}_{s i}$ 为巩膜力, $i=x, y$ 对应于巩膜力的 $X$ 和 $Y$ 方向, $F_{s}^{*}$ 定义了巩膜力的安全阈值, 若巩膜力的分 量超过此阈值, SHER 机器人的控制策略由基本导 纳控制方法切换为巩膜力约束控制。

由式(6)定义的器械速度为由一段非线性曲线 轨迹及一段定值直线轨迹组成, 如图 6 所示。其中 $\max v_{s}$ 表示器械运动速度的最大允许值, $\max \Delta F_{s}$ 定 义了器械速度由非线性曲线轨迹到定值直线轨迹的 临界点, $A 、 B$ 为非线性曲线轨迹的控制参数, 分别 取值为

$$
\left\{\begin{array}{l}
A=\max v_{\mathrm{s}} \\
B=\frac{\pi}{2 \max \Delta F_{s}}
\end{array}\right.
$$

通过试验确定 $F_{s}^{*}$ 、 $\max v_{s}$ 以及 $\max \Delta F_{s}$ 的取值 分别为 $50 \mathrm{mN} 、 5 \mathrm{~mm} / \mathrm{s}$ 以及 $20 \mathrm{mN}$ 。

器械在巩膜坐标系下的平移速度的第三项 $v_{s z}$ 决定了器械沿器械轴向的进给。在存在少量摩擦力 的情况下, 器械的轴向进给并不会对眼球组织造成 明显的作用力。因此器械的轴向进给运动应当由医 生始终进行控制, 即 $v_{s z}$ 应当由医生对机器人器械的 操作力计算得到, 如式(8)、(9)所示

$$
\begin{gathered}
\boldsymbol{F}_{s h}=\boldsymbol{A d}_{g_{s h}} \boldsymbol{F}_{h h} \\
v_{s z}=\alpha \boldsymbol{D} \boldsymbol{F}_{s h}
\end{gathered}
$$

式中, $a$ 为同式(3)中的导纳增益系数, $\boldsymbol{D}=\left[\begin{array}{lllll}0 & 0 & 1 & 0 & 0\end{array}\right.$ 0]用于提取 $\boldsymbol{F}_{s h}$ 的 $Z$ 分量, $\boldsymbol{A d}_{g_{s h}}$ 为从器械坐标系 $\{h\}$
到巩膜坐标系 $\{s\}$ 的伴随转换矩阵, 可以进一步写为

$$
\boldsymbol{A d}_{g_{s h}}=\left[\begin{array}{cc}
\boldsymbol{R}_{s h} & \hat{\boldsymbol{p}}_{s h} \boldsymbol{R}_{s h} \\
0 & \boldsymbol{R}_{s h}
\end{array}\right]
$$

式中, $\boldsymbol{R}_{s h}$ 和 $\boldsymbol{p}_{s h}$ 分别表示器械坐标系 $\{h\}$ 到机器人 坐标系 $\{s\}$ 的旋转矩阵及平移矢量, $\hat{\boldsymbol{p}}_{s h}$ 为 $\boldsymbol{p}_{s h}$ 的反对 称矩阵。

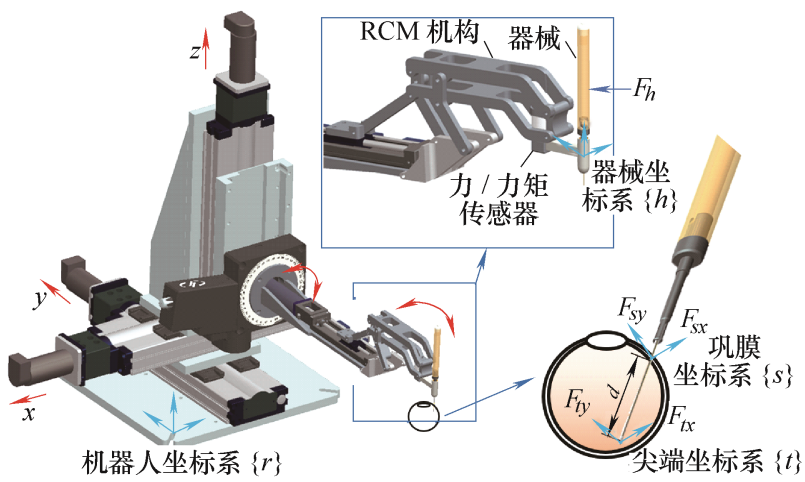

图 5 机器人坐标系示意

\section{3 尖端力约束控制}

在器械尖端力的约束控制上, 同样将尖端力作 为输入, 计算机器人未端器械在器械尖端坐标系下 的平移速度 $\left(v_{t x}, v_{t y}\right)$, 通过对其平移速度进行控制进 而抑制尖端力的大小。参考图 6 所示的非线性速度 轨迹, 可以得到平移速度 $\left(v_{t x}, v_{t y}\right)$ 的计算公式如式(11) 所示

$$
v_{t i}=\left\{\begin{array}{l}
A \sin \left(B \Delta F_{t i}\right) \quad 0<\Delta F_{t i} \leqslant \max \Delta F_{t} \\
\operatorname{sgn}\left(F_{t i}\right) \max v_{t} \quad \Delta F_{t i}>\max \Delta F_{t} \\
0 \quad \text { 其他 }
\end{array}\right.
$$

式中, $\Delta F_{t i}=\left|F_{t i}\right|-F_{t}^{*}$ 表示尖端力与安全阈值的差值, 其中 $F_{t i}$ 为尖端力, $i=x, y$ 对应于尖端力的 $X$ 和 $Y$ 方 向, $F_{t}^{*}$ 定义了尖端力的安全阈值, 若尖端力的分量 超过此阈值, SHER 机器人的控制方法由基本导纳 控制方法切换为尖端力约束控制。式中, $\max v_{t}$ 表示 器械运动速度的最大允许值, 定义了器械速度由非 线性曲线轨迹到定值直线轨迹的临界点, $A 、 B$ 为非 线性曲线轨迹的控制参数, 其取值如下所示

$$
\left\{\begin{array}{l}
A=\max v_{t} \\
B=\frac{\pi}{2 \max \Delta F_{t}}
\end{array}\right.
$$

通过试验确定 $F_{t}^{*} 、 \max v_{t}$ 以及 $\max \Delta F_{t}$ 分别取值 为 $10 \mathrm{mN} 、 2 \mathrm{~mm} / \mathrm{s}$ 以及 $10 \mathrm{mN}$ 。

末端器械在器械尖端坐标系下的平移速度的第 三项, 即沿轴向的进给速度与在巩膜坐标系下的进 给速度相同, 如式(13)所示 


$$
v_{t z}=v_{s z}
$$

在根据式(6)和式(11)得到器械尖端位置和巩膜 接触位置处的线速度后, 需要将这两处的速度转换 到同一坐标系下, 进而对机器人进行控制。由图 5 所示, 巩膜坐标系 $\{s\}$ 与尖端坐标系 $\{t\}$ 姿态相同, 两 者原点之间的距离为器械在眼内的深度 $d$, 可由力 感知器械的感知而得 ${ }^{[21]}$ 。由此, 可将尖端坐标系 $\{t\}$

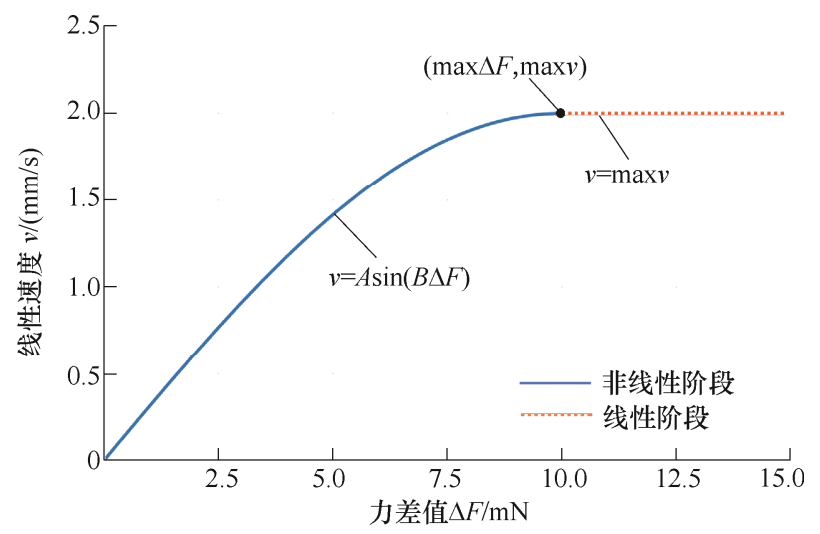

图 6 非线性速度轨迹示例

下器械尖端的线性速度 $\left(v_{t x}, v_{t y}\right)$ 转换到巩膜坐标系 $\{s\}$ 下器械绕巩膜接触点的角速度 $\left(w_{t x}, w_{t y}\right)$, 如式 (14)所示

$$
\left\{\begin{array}{l}
w_{s x}=\frac{\left(v_{t y}-v_{s y}\right)}{d} \\
w_{s y}=-\frac{\left(v_{t x}-v_{s x}\right)}{d}
\end{array}\right.
$$

式中, $d$ 为器械在眼内的深度。注意到 SHER 机器 人为 5 自由度, 因此角速度的第三项 $w_{s z}=0$ 。

至此已求得机器人末端器械在巩膜坐标系下的 全部速度分量, 如式(15)所示, 该速度即为对机器 人的约束速度

$$
\dot{\boldsymbol{x}}_{s s}=\left[\begin{array}{llllll}
v_{s x} & v_{s y} & v_{s z} & w_{s x} & w_{s y} & 0
\end{array}\right]
$$

由机器人的正运动学可求得机器人的速度雅可 比矩阵 $\boldsymbol{J}$, 进而由式(15)可进一步求出机器人的关节 速度, 用于机器人的底层控制, 如式(16)和式(17) 所示

$$
\begin{gathered}
\dot{\boldsymbol{x}}_{r s}=\boldsymbol{A} \boldsymbol{d}_{g_{r s}} \dot{\boldsymbol{x}}_{s s} \\
\dot{\boldsymbol{q}}=\boldsymbol{J}^{-1} \dot{\boldsymbol{x}}_{r s}
\end{gathered}
$$

式中, $\boldsymbol{A d}_{g_{r s}}$ 为从巩膜坐标系 $\{s\}$ 到机器人坐标系 $\{t\}$ 的伴随转换矩阵, $\boldsymbol{J}^{-1}$ 为 SHER 机器人的速度雅可 比矩阵的伪逆矩阵, $\dot{\boldsymbol{q}}$ 为机器人的关节速度, 将此 速度发送给机器人底层控制器可控制机器人按照预 设的速度轨迹进行运动, 实现对器械巩膜力和尖端
力的控制。

\section{3 视网膜血管模拟注药试验}

为验证上文所提约束控制算法的有效性, 本文 利用 SHER 手术机器人搭建了试验平台并进行了模 拟视网膜血管注药试验。试验平台如图 2 所示。除 了 SHER 机器人之外, 试验平台还包括力感知注药 针, 光纤解调仪, 以及手术显微镜等。

利用搭建的双臂机器人系统, 本文在眼球模型 上进行了模拟协同注药试验。所使用的模拟眼球如 图 7 所示，包括 3D 打印的眼眶、硅胶制成的眼球 $(\Phi=26 \mathrm{~mm})$ 以及使用硅胶管(外径 $\Phi=0.3 \mathrm{~mm}$ )及硅胶 薄膜(厚度约为 $0.1 \mathrm{~mm}$ )制成的模拟视网膜及血管。眼 球可在眼眀内自由转动, 以模拟人眼球的自由度。

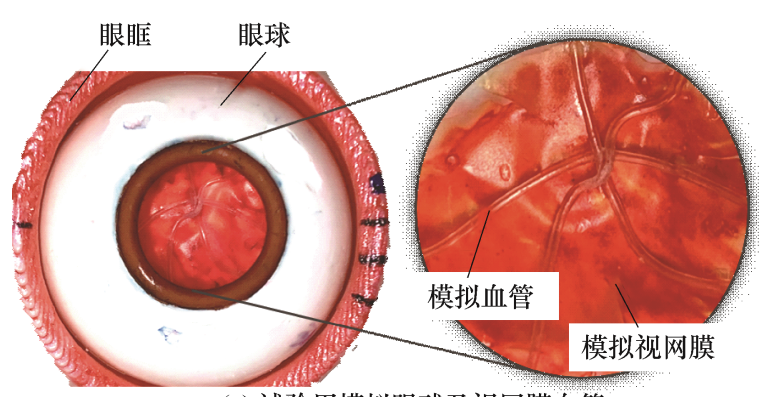

(a) 试验用模拟眼球及视网膜血管

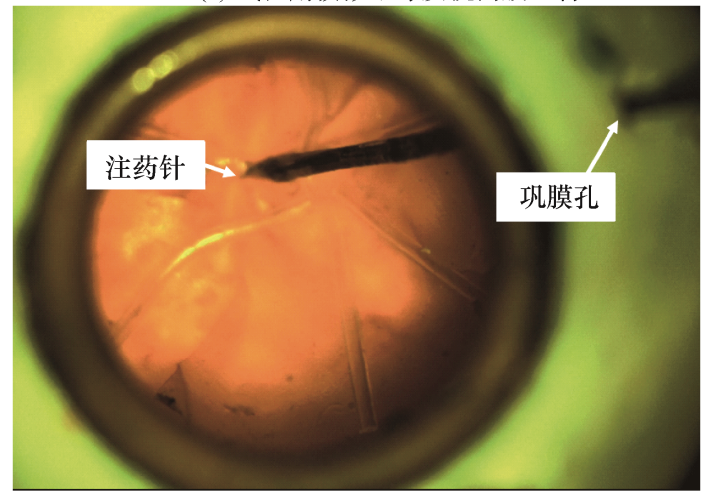

(b) 手术显微镜下器械通过巩膜孔插入眼球并刺入模拟视网膜血管

图 7 眼球模型及试验场景

为了验证所提出多点力约束的机器人控制算法 对尖端力及巩膜力的控制效果，一名试验者控制机 器人进行了模拟协同血管穿刺试验。试验步骤如下。

(1) 在式(3)所示的基本导纳控制方式下，试验 者操纵 SHER 2.0 机器人, 控制力感知注药针刺入一 眼底血管内。

(2) 切换机器人控制模式为多点力约束控制(式 (15)), 即包括巩膜力约束控制和尖端力约束控制, 该 模式将控制机器人保持手术器械上的尖端力及巩膜 力分别小于一预设的安全阈值, 本文中取巩膜力安全 
阈值 $F_{s}^{*}=50 \mathrm{mN}$, 尖端力安全阈值 $F_{t}^{*}=10 \mathrm{mN}$ 。

(3) 对眼球施加外部的扰动, 即手动以随机的 速度和方向转动眼球, 转动的最大幅度为 $10^{\circ}$, 以 产生足够大的眼球运动。

(4) 在外部扰动存在的情况下, 持续多点力约 束控制模式 $1 \mathrm{~min}$, 然后结束试验, 从眼球中撤出注 药针。

试验总计进行了 20 次, 试验中对注药针的尖端 力及巩膜力进行了记录和分析, 结果如图 8 所示。

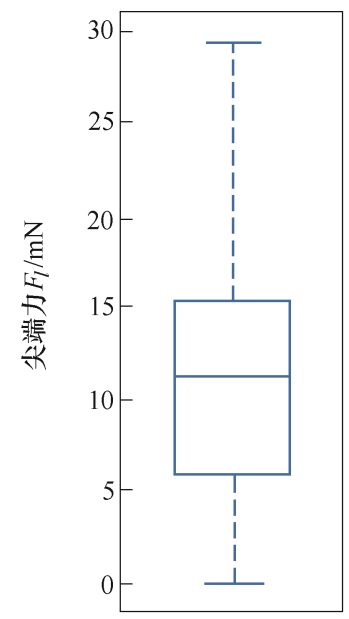

尖端力

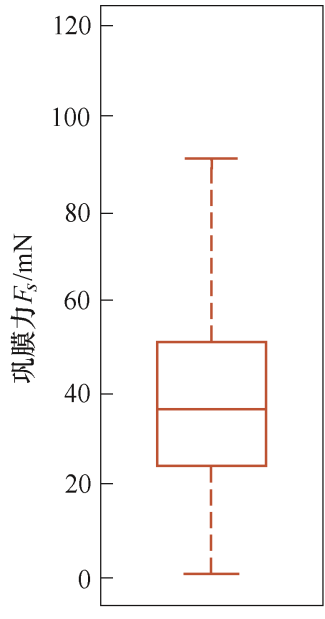

巩膜力
图 8 尖端力和巩膜力结果

经过分析, 显微注药针的平均尖端力及平均巩 膜力分别为 $10.9 \pm 6.3 \mathrm{mN}$ (土表示标准差)及 $38.1 \pm$ $20.4 \mathrm{mN}$ 。尽管试验中尖端力并没有严格被控制在预 设的安全阈值 $(10 \mathrm{mN})$ 内, 但其仍被控制在距离安全 阈值较小的范围内 $(+0.9 \mathrm{mN})$ 。另外, 在实际应用中, 通过将给定阈值调低, 可以进一步控制尖端力的值 在更小的范围内。试验中器械的巩膜力低于设定的 安全阈值 $(50 \mathrm{mN})$, 因此表明了即时在外界扰动存在 的情况下, 所提出的控制算法可以稳定的对巩膜力 进行稳定的控制。

为了进一步分析机器人相对于外界扰动的动 态响应, 本文对一例试验中机器人速度与力的变化 进行了分析, 如图 9 所示。图中上部分展示了 $X$ 方向上尖端力 $F_{t x}$ 与器械在巩膜接触处的角速度 $w_{s y}$ 的关系, 下部分展示了 $X$ 方向上巩膜力 $F_{s x}$ 与器械 在巩膜处的线速度 $v_{s x}$ 的关系。由约束控制的工作 原理可知, 当尖端力或巩膜力的值在阈值范围内 时, 多点力约束控制不被激活, 因此图中机器人的 速度响应为 0 。一旦尖端力或巩膜力的值超过阈值 后, 机器人受多点力约束控制并根据图 6 所示的 速度轨迹产生平滑的速度响应, 以抑制超过阈值的 力。由图可知, 机器人的速度响应并没有显著的突
变, 从而表明了所提出的机器人控制算法具有一定 的稳定性。
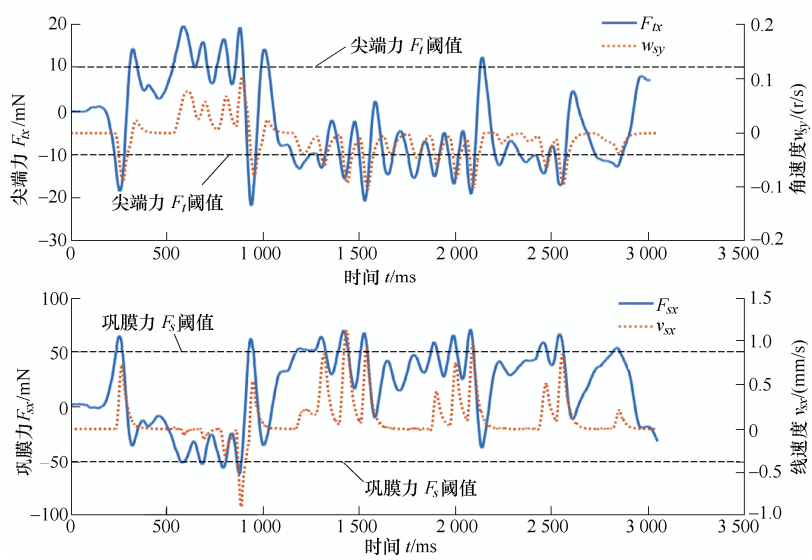

图 9 试验中机器人速度与力响应的变化关系

\section{5 结论}

（1）设计了基于多点接触力的约束控制算法, 将尖端力及巩膜力作为控制器的输入, 计算器械尖 端处及巩膜接触位置的运动速度, 并将这两处的运 动速度转换到同一坐标系下, 对机器人进行控制, 实现了对巩膜力和尖端力大小的控制。

(2) 在导纳控制中，提出并应用了一种非线性 速度规划方法, 通过一段正弦轨迹和一段直线轨迹 组成的分段曲线, 将力的输入映射为速度的输出, 并实现了速度的平滑变化。

(3) 基于 SHER 机器人和力感知手术注药器搭 建了手术机器人系统, 并制作了硅胶眼球模型以及 模拟血管, 并以此为试验平台进行了模拟血管注药 试验。试验验证了所提多点力约束机器人控制算法 对尖端力及巩膜力的抑制的有效性, 结果显示, 在 外部扰动存在的情况下, 控制算法仍然可以维持尖 端力在给定阈值附近，抑制巩膜力低于给定阈值。

(4) 提供了一种视网膜手术中器械的约束运动 控制方法, 本文的研究结果可以增强机器人在视网 膜手术中的安全性, 并进一步推进机器人在眼科手 术中的临床应用。

\section{参 考 文 献}

[1] SINGH S, RIVIERE C. Physiological tremor amplitude during retinal microsurgery[C]// Proceedings of the IEEE 28th Annual Northeast Bioengineering Conference, 2002 : 171-172.

[2] GUPTA P K, JENSEN P S, DE JUAN J E. Lecture notes in computer science[M]. Berlin: Springer-Verlag, 1999.

[3] WEISS J N. Treatment of central retinal vein occlusion by 
injection of tissue plasminogen activator into a retinal vein[J]. American Journal of Ophthalmology, 1998, 126(1): 142-144.

[4] 贺昌岩, 杨洋, 梁庆丰, 等. 机器人在眼科手术中的应 用及研究进展[J]. 机器人, 2019, 41(2): 265-275

HE Changyan, YANG Yang, LIANG Qingfeng, et al. Applications and research progress of robot assisted eye surgery[J]. Robot, 2019, 41(2): 265-275.

[5] UNERI A, BALICKI M A, HANDA J, et al. New steady-hand eye robot with micro-force sensing for vitreoretinal surgery[C]// IEEE RAS and EMBS International Conference on Biomedical Robotics and Biomechatronics. Piscataway, USA : IEEE, 2010 : 814-819.

[6] DE SMET M D, MEENINK T C, JANSSENS T, et al. Robotic assisted cannulation of occluded retinal veins[J]. PLoS One, 2016, 11(9): e0162037.

[7] GIJBELS A, POORTEN E B V, GORISSEN B, et al. Experimental validation of a robotic comanipulation and telemanipulation system for retinal surgery[C]// IEEE RAS and EMBS International Conference on Biomedical Robotics and Biomechatronics. Piscataway, USA: IEEE, 2014: 144-150.

[8] HE C Y, HUANG L, YANG Y, et al. Research and realization of a master-slave robotic system for retinal vascular bypass surgery[J]. Chinese Journal of Mechanical Engineering, 2018, 31(1): 78.

[9] NASSERI M A, EDER M, EBERTS D, et al. Kinematics and dynamics analysis of a hybrid parallel-serial micromanipulator designed for biomedical applications [C]// Proceedings of 2013 IEEE/ASME International Conference on Advanced Intelligent Mechatronics, 2013: 293-299.

[10] WEI W, POPPLEWELl C, CHANG S, et al. Enabling technology for microvascular stenting in ophthalmic surgery[J]. Journal of Medical Devices, 2010, 4(1): 167-190.

[11] RAHIMY E, WILSON J, TSAO T, et al. Robot-assisted intraocular surgery: Development of the IRISS and feasibility studies in an animal model[J]. Eye, 2013, 27(8): 972-978.

[12] UETA T, YAMAGUCHI Y, SHIRAKAWA Y, et al. Robot-assisted vitreoretinal surgery: Development of a prototype and feasibility studies in an animal model[J]. Ophthalmology, 2009, 116(8): 1538-1543.

[13] MACLACHLAN R A, BECKER B C, TABARES J C, et al. Micron: An actively stabilized handheld tool for
microsurgery[J]. IEEE Transactions on Robotics, 2012, 28(1): 195-212.

[14] HE X C, VAN GEIRT V, GEHLBACH P, et al. IRIS: Integrated robotic intraocular snake[C]// IEEE International Conference on Robotics and Automation. Piscataway, USA: IEEE, 2015: 1764-1769.

[15] HUBSCHMAN J P, BOURGES J L, CHOI W, et al. 'The Microhand': A new concept of micro-forceps for ocular robotic surgery[J]. Eye, 2010, 24(2): 364-367.

[16] IORDACHITA I, SUN Z, BALICKI M, et al. A sub-millimetric, $0.25 \mathrm{mN}$ resolution fully integrated fiber-optic force-sensing tool for retinal microsurgery[J]. International Journal of Computer Assisted Radiology and Surgery, 2009, 4(4): 383-390.

[17] XIAO J, HUANG L, SHEN L, et al. Design and research of a robotic aided system for retinal vascular bypass surgery[J]. Journal of Medical Devices, 2014, 8(4): 044501-1-044501-6.

[18] EBRAHIMI A, PATEL N, HE C, et al. Adaptive control of sclera force and insertion depth for safe robot-assisted retinal surgery $[\mathrm{C}] / /$ Proceedings of IEEE International Conference on Robotics and Automation (ICRA), 2019: 9073-9079.

[19] EBRAHIMI A, HE C, ROIZENBLATT $M$, et al. Real-time sclera force feedback for enabling safe robot-assisted vitreoretinal surgery[C]// Proceedings of 2018 40th Annual International Conference of the IEEE Engineering in Medicine and Biology Society (EMBC), 2018: 3650-3655.

[20] HE X, BALICKI M, GEHLBACH P, et al. A multi-function force sensing instrument for variable admittance robot control in retinal microsurgery[C]// 2014 IEEE International Conference on Robotics and Automation (ICRA). IEEE, 2014: 1411-1418.

[21] HE C, YANG E, IORDACHITA I. Dual-stiffness force-sensing cannulation tool for retinal microsurgery [C]// Proceedings of 2019 41st Annual International Conference of the IEEE Engineering in Medicine and Biology Society (EMBC), Berlin, Germany, 2019: 3212-3216.

作者简介: 贺昌岩, 男, 1993 年出生, 博士研究生。主要研究方向为医 疗机器人。

E-mail: changyanhe@buaa.edu.cn

杨洋(通信作者), 男, 1962 年出生, 博士, 教授, 博士研究生导师。主 要研究方向为智能机械设计、医疗机器人、多指灵巧手。

E-mail: yang_mech@buaa.edu.cn 